\title{
Apresentação clínica da Doença de Creutzfeldt-Jakob como Síndrome Cerebelar
}

\author{
Clinical presentation of Creutzfeldt-Jakob Disease as Cerebellar Syndrome
}

\section{Ideli Neitzke', Henrique Ferreira de Brito' ', Aline Brandão', Janaína Luz Narciso-Schiavon², Leonardo de Lucca Schiavon², Fernando da Costa Buzzoleti ${ }^{3}$}

\begin{abstract}
RESUMO
A doença de Creutzfeldt-Jakob (DCJ) constitui-se na mais comum das doenças priônicas em seres humanos. Apresentamos um caso de DCJ tipo esporádico em um homem de 48 anos, que iniciou quadro de síndrome cerebelar, cuja clínica evoluiu compatível com DCJ associado à alteração do eletroencefalograma (EEG) típica e líquor (LCR) com alteração da proteína 14-3-3. São discutidosos métodos diagnósticos, as expectativas sobrea identificação de fatores de transmissão e a terapêutica atual.
\end{abstract}

Unitermos: Doença de Creutzfeldt-Jakob. Demência. Doenças de Príon.

Citação: Neitzke I, Brito HF, Brandão A, Narciso-Schiavon JL, Schiavon LL, Buzzoleti FC. Apresentação clínica da Doença de Creutzfeldt-Jakob como Síndrome Cerebelar.

\section{SUMMARY}

Creutzfeldt-Jakob disease (CJD) is the most frequent prion disease in humans. A case of sporadic form of CJD is reported. A 48 year-old male presented with cerebellar syndrome compatible with CJD associated with typical electroencephalogram modification and alteration in 14-3-3 protein in cerebrospinal fluid (CSF). The clinical characteristics, laboratory findings, differential diagnosis, mechanisms of transmission and the actual therapeutic approach are discussed.

Keywords: Creutzfeldt-Jakob Syndrome. Dementia. Prion Diseases.

Citation: Neitzke I, Brito HF, Brandão A, Narciso-Schiavon JL, Schiavon LL, Buzzoleti FC. Clinical presentation of CreutzfeldtJakob Disease as Cerebellar Syndrome.
Trabalho realizado no Serviço de Clínica Médica, Hospital Heliópolis, São Paulo, SP.

1. Médico Residente do Serviço de Clínica Médica, Hospital Heliópolis, São Paulo, SP.

2. Médico do Serviço de Clínica Médica, Hospital Heliópolis, São Paulo, SP.

3. Chefe do Serviço de Clínica Médica, Hospital Heliópolis, São Paulo, SP.
Endereço para correspondência: Janaína L Narciso-Schiavon R. Cônego Xavier $276,4^{\circ}$ andar 04231-902 São Paulo, SP Fone/fax: (55 11) 2274-7600 ramal 103 E-mail: janaina.narciso@uol.com.br

Recebido em: 12/09/07 Revisado em: 13/09/07 a 28/11/07 Aceito em: 29/11/07

Conflito de interesses: não 


\section{INTRODUÇÃO}

A doença de Creutzfeldt-Jakob (DCJ) é a mais freqüentedasdoenças priônicashumanas-grupode doenças raras referidas como encefalopatias espongiformes ${ }^{1,2}$. Constitui-se de um quadro de demência rapidamente progressiva, com sinais piramidais, extra-piramidais, cerebelares ealterações típicas doeletroencefalograma ${ }^{3}$.Apresentaincidênciade 0,4-2,5/1 milhão de habitantes/ano, idade média de 60 anos e distribuição semelhante entre homens e mulheres ${ }^{4}$.

\section{Relato de Caso}

Um homem branco de 48 anos, natural e residente de São Paulo, procurou atendimento médico poralteraçãoda marcha edificuldade para movimentação de dimídio esquerdo. O paciente referia início progressivo há 15 dias de dificuldade para deambulação, associada à rigidez e incoordenação do membrosuperioresquerdo.Previamentehígido,nãousava medicações, negava etilismo ou uso de drogasilícitas bem como antecedentes familiares significativos.

O exame físico geral da entrada foi normal e ao exame neurológico paciente se encontrava vigil, consciente, orientado no tempo e espaço. Apresentava hipertonia nos membros superior e inferior esquerdos. A força muscular e os reflexos tendinosos encontravam-se normais globalmente. A sensibilidade estava preservada e constatou-se ausência de sinais meningorradiculares. A marcha era atáxica e as provas indicador-nariz e calcanhar-joelho foram positivas. Havia dismetria e decomposição do movimento em membro superior esquerdo.

Durante o exame o paciente desenvolveu espontaneamenteposturaemflexãodepunhoesquerdocommãoemgarra, sugerindoquadrodedistonia. Não havia alterações em pares cranianos. Foi feito diagnóstico sindrômico principal de síndrome cerebelar, embora a presença de hipertonia e distonia comreflexosnormaissugerissemumquadroextrapiramidal. Os exames laboratoriais iniciais mostravam eletrólitos normais, incluindo cálcio, hemograma normal, sem macrocitose, TSH normal, sorologia para HIV e VDRL negativos. A tomografia computadorizada (TC) e a ressonância nuclear magnética (RNM) de encéfalo foram normais. O exame do líquor foi normal a não ser por discreta hiperproteinorraquia ( $59 \mathrm{mg} / \mathrm{dL}$ ). A pesquisa pela tinta da China e o VDRL do líquor foram negativos. A dosagem ceruloplasmina sérica foi normal.

Durante a investigação, o paciente evoluiu comgeneralizaçãodoquadro,miocloniaseacentua- ção dos episódios de distonia. Foi iniciado tratamento empírico para meningoencefalite herpética com aciclovir. Realizado eletroencefalograma (EEG), que mostroudescargasagudasrepetidasdealtavoltagem trifásicas (Figura 1). Apesar do tratamento antiviral, o paciente evoluiu rapidamente com piora das mioclonias, rebaixamento do nível de consciência e episódios de crises convulsivas focais. Devido ao rebaixamento do nível de consciência foi realizada intubação orotraqueal e iniciada sedação contínua com midazolam e fentanil.

Nova TC de crânio foi normal o líquor mantinha o mesmo padrão observado na entrada. Durante várias tentativas de desmame da sedação, houve pioradasmiocloniasefoiobservadoreflexocutâneoplantar em retirada bilateralmente. Devido ao quadro clínico sugestivo e ao EEG típico, foi feita hipótese de doença priônica. A citogenética para doença priônica tipo familiar foi negativa e proteína 14-3-3 nolíquor foi positiva. Após início de tratamento com clonazepam e ácido valpróico houve melhora parcial das mioclonias. Apesar do suporte clínico, houve piora progressiva do quadro com evolução para óbito após cinco meses do início dos sintomas. Os achados necroscópicos confirmaram a hipótese de doença priônica.

\section{DISCUSSÃO}

DCJ é a mais freqüente das doenças priônicas humanasapresentandoquatrosubtipos:1.Esporádico $(87 \%) ; 2$. Familiar (10\%) autossômica dominante; 3. latrogênica transmitida por tecidos humanos em casos de transplante de córnea, enxertos de duramáter, infusão dehormônio de crescimento retirado

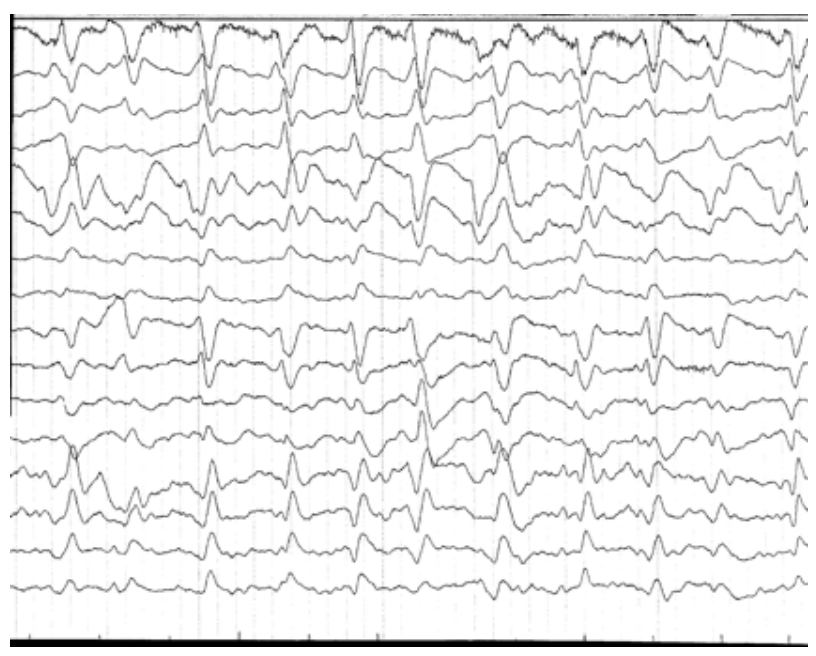

Figura 1. Eletroencefalograma com alterações típicas da Doença de Creutzfeldt-Jakob. 
de hipófise humana etc.; e 4. DCJ nova variante relacionada com epidemia de encefalopatia espongiforme bovina ${ }^{2,5,6}$.

Oagenteinfecciosoenvolvidoéchamadopríon, umaanormalidadeestruturaldeproteína priônicacelular, de função biológica desconhecida, encontrada nos tecidos saudáveis ${ }^{7}$. Logo, a DCJ é devida a degeneração neuronal resultante do acúmulo da isoforma patológica (PrPSc) da proteína priônica celular normal (PrPc). Com a estrutura reformulada, a proteína interrompe a função e determina a morte neuronal ${ }^{8}$.

Curso típico de invariável progressão, com mioclonias e piora das habilidades cognitivas em questão de semanas a dias. Pode apresentar pródromos de astenia, hiporexia e alterações comportamentais. Cerca de um terço dos pacientes inicia com sinaisfocais, ataxia, afasia, perda visual, hemiparesia, amiotrofia, mioclonias, postura rígida. Evolui com mutismo acinético e morte em $100 \%$ dos $\operatorname{casos}^{9,10}$.

O diagnóstico é suspeitado pelo quadro de demênciarapidamenteprogressivanatotalidadedos casos. Não há marcador laboratorial para a DCJ. O eletroencefalograma (EEG) mostra-se alterado na grande maioria dos pacientes, independentemente da variedade e da fase da doença. Classicamente, apresentacomplexosperiódicosdealtavoltagemem intervalos de 0,5-2 segundos (ondas trifásicas). Precocemente, apresenta ritmo de baselento, difuso ou lateralizado, com descargas epileptiformes (responsáveis pelas mioclonias). Constitui-se em um exame nãoinvasivodegrandeimportânciadiagnóstica,com especificidade de $86 \%$ e sensibilidade de $67 \%$. Essas alterações, no entanto, podem ocorrer nos casos de doença de Alzheimer, abscessos cerebrais, encefalopatia metabólica, leucoencefalopatia progressiva multifocal e intoxicação por bismuto e lítio ${ }^{11,12}$.

A análise do líquido cefalorraquidiano (LCR) freqüentemente é normal, sendo útil para exclusão de encefalites e neuroinfecção. $O$ teste por Western Blot para fragmentos da proteína 14-3-3 no LCR chega a ter uma sensibilidade de $96 \%$ e especificidade de $99 \%$ e, quando positivo, no contexto clínico apropriado,éaltamentesugestivodedoença priônica esporádica.Podeestarpositivoaindana encefalopatiaherpética,hemorragiasubaracnóide,encefalopatia paraneoplásica ${ }^{8,12,13}$.

Estudos de neuroimagem (RNM) também são importantes no diagnóstico da DCJ e principalmente no diagnóstico diferencial. Na forma esporádica, a RNM pode ser normal ou apresentar apenas atrofia cerebral. Em raros casos apresenta hipersinal nos gânglios da base e no córtex. Na forma recém descrita de Nova Variante, éaltamente sensível eespecífica quandodemonstrahipersinal na regiãotalâmica posterior chamado sinal pulvinar ${ }^{12,14-16}$.

A biópsia de tonsila palatina pode evidenciar a presença de proteína priônica anormal. Tem importância apenas na forma nova variante, que pode acometer, além das tonsilas, tecido linfonodal e baço ${ }^{16}$. A forma iatrogênica é diagnosticada por meio de quadro clínico típico e história relevante de fator de risco 9 . O diagnóstico definitivo depende da análise neuropatológica e neuro-histoquímica. São característicaspatognomônicas, aespongiose,perda neuronal e gliose ${ }^{17}$. O diagnóstico diferencial inclui neuroinfecções como sífilis, herpes simples, toxinas como lítio, bismuto, encefalopatia de Hashimoto, doença de Whipple e meningite carcinomatosa ${ }^{18}$.

Apesardeváriosesquemasterapêuticosterem sido estudados, nenhum até o momento foi efetivo em mudar a evolução fatal da doença. Dentre as terapias relatadas, encontram-se drogas antivirais (vidarabina, aciclovir, amantadina, idoxuridina), clorpromazina, polissulfatodepentosam, interferon, anticonvulsionantes e corticóides ${ }^{1,10,19}$. Aproximadamente $90 \%$ dos indivíduos acometidos evoluem para óbito em um ano. Atualmente o tratamento recomendadoébasicamentedesuporteecontroledas complicações.

\section{CONCLUSÃO}

Em conclusão, a DCJ constitui diagnóstico raro que deve ser suspeitado em casos de demência rapidamente progressiva associado a alterações cerebelares, piramidais ou extrapiramidais. Ainda não há tratamento capaz de modificar a evolução fatal da doença, no entanto, o desenvolvimento de técnicas que permitam um diagnóstico mais precoce pode possibilitar o estudo de medidas terapêuticas eficazes no futuro.

\section{REFERÊNCIAS BIBLIOGRÁFICAS}

1. Tyler KL. Creutzfeldt-Jakob Disease. N Engl J Med 2003;348:681.

2. Araújo $A B Q$, Oliveira ALA. Doenças por prions. Rev Neurocienc 1998;6:33-46.

3. Prusiner SB. Shattuck lecture: Neurodegenerative diseases and prions. N Engl J Med 2001;344:1516-26.

4. Brown P, Gibbs CJ Jr, Rodgers-Johnson P, Asher DM, Sulima MP, Bacote $A$, et al. Human spongiform encephalopathy: the National Institutes of Heatlh series of 300 cases of experimentally transmited disease. Ann Neurol 1994;35:513-29.

5. Zeidler M, Stewart GE, Barraclough CR, Bateman DE, Bates D, Burn DJ, et al. New variant Creutzfeldt-Jakob disease: neurological features and diagnosis tests. Lancet 1997;350:930-7.

6. Epstein LE, Brown P. Bovine spongiform encephalopathy and a new variant of Creutzfeldt Jakob disease. Neurology 1997;48:569-71. 
7. Chiarini LB, Freitas ARO, Zanta SM, Brentani RR, Martins VR, Linden R. Celular prion protein transduces neuroprotective signals. EMBO 2002;21:3317-26.

8. Huang N, Marie SK, Livramento JA, Chammas R, Nitrini R. 143-3 protein in the CSF of patients with rapidly progressive dementia. Neurology 2003:61;354-1.

9. Brandel JP, Delasnerie-Lauprêtre N, Laplanche JL, Hauw JJ, Alpérovitch A. Diagnosis of Creutzfeldt Jakob disease. Neurology 2000;54:1095-9.

10. Kovacs T, Arányi Z, Szirmai I, Lantos PL. Creutzfeldt Jakob disease with amyotrophy and demyelinating polyneuropathy. Arch Neurol 2002;59:1811-4.

11. Steinhoff BJ, Rãcker S, Herrendorf G, Poser S, Grosche S, Zerr I, et al. Accuracy and reability of periodic sharp wave complexes in Creutzfeldt-Jakob disease. Arch Neurol 1996;53:162-6.

12. Moretti R, Torre P, Nasuelli D, Ferretti G, Antonello RM, Povolato $M$, et al. Diagnostic instruments for Creutzfeldt Jakob disease. Eur J Neurol 2000;7:363-4

13. Hsich G, Kenney K, Gibbs CJ, Lee KH, Harrington MG. The 14-3-3 brain protein in cerebrospinal fluid as a marker for transmissible spongiform encephalopathies. N Engl J Med 1996;335:924-30.

14. Krovanen J, ErkinJuntti T, Ivanaimen M. Cerebral MR and CT imaging in Creutzfeldt-Jakob disease. J Comput Assist Tomogr 1985;9:125-8.

15. Bahn MM, Parchi P. Abnormal diffusion-weighted magnetic resonance images in Creutzfeldt-Jakob disease. Arch Neurol 1999;56:577-83.

16. Hill AF, Butterworth RJ. Joiner S, Jackson G, Rossor MN, Thomas DJ, et al. Investigation of variant Creutzfeldt-Jakob disease and other human prion diseases with tonsill biopsy samples. Lancet 1999;353:183-4.

17. Will RG, Cousens SN, Farrington CO, Smith PG, Knight RSG, Ironside JW. Deaths from variant Creutzfeldt Jakob disease. Lancet 1999;353:979.

18. Poser S, Mollenhauer B, Kraub A, Zerr I Steinhoff BJ, Schroeter A, et al. How to improve the clinical diagnosis of Creutzfeldt-Jakob disease. Brain 1999;122:2345-51.

19. Brown P. Drug therapy in human and experimental transmissible spongiform encephalopathy. Neurology 2002;58:1720-5. 